Muro de la Investigación, 2018: 3(1), Enero-Julio, ISSN: $2523-2886$

DOI:https://doi.org/10.17162/rmi.v3i1.1111

\title{
Desempeño docente y rendimiento académico de los estudiantes del curso de técnicas de estudio de una universidad privada confesional
}

\author{
Betsabeth Teresa Padilla Macedo ${ }^{1 a}$ y Avelino Sebastián Villafuerte De la Cruz ${ }^{2}$ \\ Universidad Peruana Unión, Perú ${ }^{12}$
}

\section{Recibido: 02 de abril de 2018}

Aceptado: 11 de noviembre de 2018

\begin{abstract}
Resumen
El objetivo fue determinar si existe relación significativa entre la percepción del desempeño docente y el rendimiento académico de los estudiantes del curso de técnicas de estudio de una universidad privada confesional. La metodología fue de tipo cuantitativo correlacional, corte transversal, el diseño fue no experimental. La muestra fue de 287 estudiantes de las diferentes escuelas profesionales de la universidad. Para evaluar el desempeño docente se utilizó como instrumento la "Encuesta de Desempeño Docente" elaborada por Palomino (2012), validada y fiable $(\alpha=.941)$. Para el rendimiento académico se emplearon los promedios obtenidos en el curso de técnicas de estudio. Se hizo un análisis Correlación de Spearman. Se identificó que existe relación significativa entre la percepción del desempeño docente y el rendimiento académico $(\mathrm{r}=0.453)$. Asimismo, se identificó que existe relación significativa entre la percepción de estrategias didácticas y el aprendizaje de los estudiantes $(\mathrm{r}=0.404)$; entre la percepción de materiales didácticos y el aprendizaje de los estudiantes $(\mathrm{r}=0.404)$; entre la percepción de capacidades pedagógicas y el aprendizaje de los estudiantes $(\mathrm{r}=0.442)$; entre la percepción de responsabilidad del docente y el aprendizaje de los estudiantes $(r=0.447)$. El 42.9 $\%$ de estudiantes de psicología y el $37.1 \%$ de estudiantes de contabilidad considera que el desempeño del docente del curso de técnicas de estudio es alto, lo cual se ve reflejado en que el $65.5 \%$ de los estudiantes tiene un promedio superior a 15 . Se concluye que a mayor desempeño del docente mayor rendimiento académico de los estudiantes del curso de técnicas de estudio.
\end{abstract}

Palabras clave: desempeño docente; rendimiento académico; estrategias didácticas; materiales didácticos; capacidades padagógicas; responsabilidad docente.

\section{Teaching performance and academic performance of the students of the study techniques course of a private confessional university}

\footnotetext{
${ }^{a}$ Correspondencia al autor

E-mail: padilla@upeu.edu.pe
} 


\begin{abstract}
The objective of this study was to determine if there is a significant relationship between the perception of the teaching performance and the academic performance of the students of the study techniques course in a private confessional university. The methodology was quantitative and correlational, using a cross section, and the design was not experimental. The sample was made up of 287 students of the different professional schools of the university. To evaluate the teaching performance, the "Teacher Performance Survey" developed by Palomino (2012), previous shown to be validated and reliable $(\alpha=.941)$, was used as an instrument. For the academic performance, the averages obtained in the study techniques course were used. A Spearman correlation analysis was made. A significant relationship was identified between the perception of teacher performance and academic performance $(r=0.453)$. Likewise, a significant relationship was identified between the perception of didactic strategies and student learning $(\mathrm{r}=0.404)$; between the perception of didactic materials and student learning $(\mathrm{r}=$ $0.404)$; between the perception of pedagogical abilities and student learning $(\mathrm{r}=0.442)$; between the perception of teacher responsibility and student learning $(r=0.447) .42 .9 \%$ of psychology students and $37.1 \%$ of accounting students consider that the teacher's performance of the study techniques course is high, which is reflected in the fact that $65.5 \%$ of students have an average higher than 15 on a 20 point scale. It is concluded that the higher the teacher's performance, the higher the academic performance of the students of the study techniques course.
\end{abstract}

Key words: Teaching performance; academic performance; teaching strategies; didactic materials; pedagogical abilities; teaching responsibility.

\title{
Introducción
}

Los cambios a los que se encuentra sometida la sociedad educativa están demandando un cambio urgente de actuación de las universidades, de los docentes y del resto de interlocutores de la comunidad educativa (Ortega, 2010).

De igual importancia, Alterio Ariola \& Pérez Loyo, (2009) afirman que la calidad de una universidad depende de la formación que alcancen sus estudiantes, esto se logra mediante ciertos lineamientos como: investigación, infraestructura, capacitación y el perfeccionamiento docente. El perfeccionamiento de este, permitirá fortalecer sus competencias, alcanzando un mejor desempeño en el aula.

De ahí que, Palomino Zamudio (2012), Ramírez Sánchez (2014), Fernandes, Sotolongo, Ii, Cristóbal, \& Martínez (2016), mencionan que hoy en día es valioso "el estudio del desempeño de los docentes puesto que permite establecer si la calidad de la educación que brindamos en las universidades es congruente con los planteamientos y estándares educativos planteados". A la par, permite tomar decisiones en favor de la calidad, tanto del desempeño de los docentes como del proceso de enseñanza-aprendizaje. 
Por otra parte, para Garbanzo (2007), el aprendizaje que alcanzan los estudiantes "se mide a través del rendimiento académico, el cual es un indicador que permite un acercamiento a la realidad educativa". Además, Ortiz, Ortiz, Machado, \& Frutos, (2014) señalan que "el rendimiento académico expresa el grado de logro que han tenido los estudiantes en el aprovechamiento y comprensión de los contenidos docentes"; en otras palabras, lo que el alumno ha asimilado como resultado de un proceso de formación, definiendo de alguna forma el éxito o fracaso en el estudio.

Asimismo, Garbanzo (2007) señala que los estudios del rendimiento académico en la educación superior son aún más valiosos en la coyuntura mundial actual, debido a la actividad que experimenta el sector universitario en el marco de una sociedad caracterizada por el rápido avance del conocimiento, la sencillez en la transmisión de la información y los cambios en las organizaciones sociales.

Aparicio et al., (2011), argumenta que el rendimiento académico es transcendental, aunque depende de elementos inherentes tanto a asuntos personales (procedencia, edad, género, si trabaja o no) como al situación en la que se desarrollan los procesos de enseñanza y aprendizaje (el entorno, el desarrollo de la clase y la materia), el papel del docente es decisivo, por su parte Ortiz et al., (2014), refiere que el bajo rendimiento académico está ligado a variables académicas y propios del alumno. "Dentro de las pedagógicas se consideran las expectativas y actitudes del profesor, su formación y experiencia, lo que también podría llamarse desempeño docente. Respecto a las variables propias del alumno, se han estudiado las sociodemográficas, familiares, motivacionales, cognoscitivas y emocionales".

El curso de técnicas de estudio es de importancia para la formación de capacidades de investigación y actitudes hacia el aprendizaje, si el estudiante recibe una buena base en esa materia tiene asegurado en gran parte el éxito de su vida universitaria. Potenciando sus habilidades y mejorando sus procesos de aprendizaje. De aquí la importancia de un buen desempeño docente, pues esto podría afectar el rendimiento del estudiante.

La investigación del presente trabajo se orientada a analizar el desempeño de los docentes del curso de Técnicas de Estudio de una universidad privada confesional, a la vez determinar si esto se relaciona con el aprendizaje de los estudiantes.

Finalmente, ante lo anteriormente argumentado, la presente investigación responderá la pregunta: ¿Existe relación significativa entre la percepción del desempeño docente y el rendimiento académico de los estudiantes del curso de técnicas de estudio de una universidad privada confesional, 2018 ? 


\section{Metodología}

\subsection{Diseño de investigación}

La presente investigación posee un enfoque cuantitativo, según Hernández, Fernández, \& Baptista (2010) “usa la recolección de datos para probar hipótesis, con base en la medición numérica y el análisis estadístico, para probar teorías". La investigación es de tipo descriptivo correlacional, de corte transversal. El diseño es no experimental.

\subsection{Población y muestra}

La población de estudio de esta investigación son los estudiantes del curso de Técnicas de Estudio del primer año de una universidad privada confesional. Esta población está compuesta por alumnos varones y mujeres de diferentes especialidades. Para el semestre académico 2018 I se contó con 287 estudiantes matriculados. La muestra es probabilística, está representada por estudiantes de las siete Escuelas Profesionales de las tres facultades que conforman esta universidad.

\subsection{Instrumentos}

2.3.1. Desempeño académico

El instrumento a utilizar será la "Encuesta de Desempeño Docente" instrumento elaborado por Palomino (2012), validado en la Universidad Nacional Mayor de San Marcos, el cual realizó un análisis para confirmar la fiabilidad y validez de las escalas usadas. En el caso de la fiabilidad, se usó el valor Alfa de Cronbach $(\alpha=.941)$, el cual fue alto para medir la consistencia interna de las escalas. Las alternativas tienen una escala tipo Likert, con seis alternativas de respuesta.

\subsubsection{Rendimiento académico}

Para la obtención de estos valores se consideró como referencia el promedio de notas del curso de Técnicas de Estudio de las diferentes Escuelas Profesionales.

\subsection{Análisis y procesamiento de datos}

Se tabularon los datos en el software estadístico SSPS 23.0. Se realizó un análisis descriptivo para las preguntas de datos generales. Para analizar la correlación de las variables se realizó un análisis de Correlación de Spearman. 
Se utilizó la tabla de correlaciones propuesta por Martínez Rebollar \& Campos Francisco (2015).

Tabla 1

Niveles de relación ( $\mathrm{r}$ )

\begin{tabular}{cc}
\hline Valor de rho & Significado \\
\hline-1 & Correlación negativa grande y perfecta \\
-0.9 a -0.99 & Correlación negativa muy alta \\
-0.7 a -0.89 & Correlación negativa alta \\
-0.4 a -0.69 & Correlación negativa moderada \\
-0.2 a -0.39 & Correlación negativa baja \\
-0.01 a -0.19 & Correlación negativa muy baja \\
0 & Correlación nula \\
0.01 a 0.19 & Correlación positiva muy baja \\
0.2 a 0.39 & Correlación positiva baja \\
0.4 a 0.69 & Correlación positiva moderada \\
0.7 a 0.89 & Correlación positiva alta \\
0.9 a 0.99 & Correlación positiva muy alta \\
1 & Correlación positiva grande y perfecta
\end{tabular}

Fuente: Martínez Rebollar \& Campos Francisco (2015)

\section{Resultados} resultados.

De acuerdo a los análisis realizados en la investigación, se presentan los siguientes

\section{Desempeño docente}

Tabla 2

Distribución de las escuelas profesionales según niveles del desempeño docente

\begin{tabular}{lcccccccc}
\hline $\begin{array}{l}\text { Desempeño } \\
\text { docente }\end{array}$ & $\begin{array}{c}\text { Ingeniería } \\
\text { ambiental }\end{array}$ & $\begin{array}{c}\text { Ingeniería } \\
\text { de } \\
\text { sistemas }\end{array}$ & Arquitectura & Psicología & Marketing & Administración & Contabilidad & Total \\
\hline Bajo & $9(32.1)$ & $7(35)$ & $10(41.7)$ & $2(28.6)$ & $8(38.1)$ & $13(43.3)$ & $4(11.4)$ & $\begin{array}{c}53 \\
(32.1)\end{array}$ \\
Medio & $11(39.3)$ & $8(40)$ & $9(37.5)$ & $2(28.6)$ & $6(28.6)$ & $9(30)$ & $18(51.4)$ & 63 \\
$(38.2)$ \\
Alto & $8(28.6)$ & $5(25)$ & $5(20.8)$ & $3(42.9)$ & $7(33.3)$ & $8(26.7)$ & $13(37.1)$ & $\begin{array}{c}49 \\
(29.7)\end{array}$ \\
Total & $28(100)$ & $20(100)$ & $24(100)$ & $7(100)$ & $21(100)$ & $30(100)$ & $35(100)$ & 165 \\
$(100)$
\end{tabular}


Fuente: Elaboración propia

En la tabla 2, observamos que los estudiantes de la escuela de psicología (42.9\%) y contabilidad (37.1\%) perciben que el desempeño del docente de Técnicas de Estudio es de alto nivel. Vale precisar que los estudiantes de las escuelas de Ingeniería ambiental (39.3\%), Ingeniería de sistemas (40\%) y Contabilidad (51.4\%) perciben un nivel medio de desempeño docente. No obstante, en la escuela de Administración (43.3\%), los estudiantes perciben que el docente tiene un nivel bajo de desempeño docente.

Tabla 3

Niveles de las dimensiones de desempeño docente

\begin{tabular}{|c|c|c|c|c|c|c|c|c|c|}
\hline & Nivel & $\begin{array}{l}\text { Ingeniería } \\
\text { ambiental }\end{array}$ & $\begin{array}{l}\text { Ingeniería } \\
\text { sistemas }\end{array}$ & Arquitectura & Psicología & Marketing & Adm. & Cont. & Total \\
\hline \multirow{4}{*}{$\begin{array}{c}\text { Estrategias } \\
\text { didácticas }\end{array}$} & Bajo & $7(25)$ & $6(30)$ & $13(54.2)$ & $2(28.6)$ & $8(38.1)$ & $14(46.7)$ & $6(17.1)$ & $56(33.9)$ \\
\hline & Medio & $11(39.3)$ & $10(50)$ & $8(33.3)$ & $3(42.9)$ & $7(33.3)$ & $7(23.3)$ & $14(40)$ & $60(36.4)$ \\
\hline & Alto & $10(35.7)$ & $4(20)$ & $3(12.5)$ & $2(28.6)$ & $6(28.6)$ & $9(30)$ & $\begin{array}{c}15 \\
(42.9)\end{array}$ & 49 (29.7) \\
\hline & Total & $28(100)$ & $20(100)$ & $24(100)$ & $7(100)$ & $21(100)$ & $30(100)$ & $\begin{array}{c}35 \\
(100)\end{array}$ & $165(100)$ \\
\hline \multirow{4}{*}{$\begin{array}{l}\text { Materiales } \\
\text { didácticos }\end{array}$} & Bajo & $7(25)$ & $7(35)$ & $6(25)$ & $2(28.6)$ & $8(38.1)$ & $14(46.7)$ & $8(22.9)$ & $52(31.5)$ \\
\hline & Medio & $15(53.6)$ & $8(45)$ & $9(37.5)$ & $3(42.9)$ & $6(28.6)$ & $8(26.7)$ & $\begin{array}{c}17 \\
(48.6)\end{array}$ & $66(40)$ \\
\hline & Alto & $6(21.6)$ & $5(20)$ & $9(37.5)$ & $2(28.6)$ & $7(33.3)$ & $8(26.7)$ & $\begin{array}{c}10 \\
(28.6)\end{array}$ & $47(28.5)$ \\
\hline & Total & $28(100)$ & $20(100)$ & $24(100)$ & $7(100)$ & $21(100)$ & $30(100)$ & $\begin{array}{c}35 \\
(100)\end{array}$ & $165(100)$ \\
\hline \multirow{4}{*}{$\begin{array}{l}\text { Capacidades } \\
\text { pedagógicas }\end{array}$} & Bajo & $11(39.3)$ & $7(35)$ & $14(58.3)$ & $2(28.6)$ & $9(42.9)$ & $13(43.3)$ & $5(14.3)$ & $61(37)$ \\
\hline & Medio & $9(32.1)$ & $9(45)$ & $6(25)$ & $3(42.9)$ & $5(23.8)$ & $11(36.7)$ & $\begin{array}{c}13 \\
(37.1)\end{array}$ & $56(33.9)$ \\
\hline & Alto & $8(28.6)$ & $4(20)$ & $4(16)$ & $2(28.6)$ & $7(33.3)$ & $6(20)$ & $\begin{array}{c}17 \\
(48.1)\end{array}$ & $48(29.1)$ \\
\hline & Total & $28(100)$ & $20(100)$ & $24(100)$ & $7(100)$ & $21(100)$ & $30(100)$ & $\begin{array}{c}35 \\
(100)\end{array}$ & $165(100)$ \\
\hline \multirow{4}{*}{$\begin{array}{l}\text { Responsabil } \\
\text { idad en el } \\
\text { desempeño }\end{array}$} & Bajo & $8(28.6)$ & $5(25)$ & $11(45.8)$ & $2(28.6)$ & $6(28.6)$ & $12(40)$ & $5(14.3)$ & 49 (29.7) \\
\hline & Medio & $15(53.6)$ & $13(65)$ & $8(33.3)$ & $3(42.9)$ & $8(38.1)$ & $13(43.3)$ & $\begin{array}{c}15 \\
(42.9)\end{array}$ & $75(45.5)$ \\
\hline & Alto & $5(17.6)$ & $2(10)$ & $5(20.8)$ & $2(28.6)$ & $7(33.3)$ & $5(16.7)$ & $\begin{array}{c}15 \\
(42.9)\end{array}$ & $41(24.8)$ \\
\hline & Total & $28(100)$ & $20(100)$ & $24(100)$ & $7(100)$ & $21(100)$ & $30(100)$ & $\begin{array}{c}35 \\
(100)\end{array}$ & $165(100)$ \\
\hline
\end{tabular}

Fuente: Elaboración propia. 
En la tabla 03, respecto a la dimensión Estrategias didácticas, los estudiantes de contabilidad (42.9\%) e ingeniería ambiental (35.7\%) perciben un alto nivel de desempeño docente. Por su parte, los estudiantes de ingeniería de sistemas (50\%) y psicología (42.9\%) presentan un nivel medio. No obstante, arquitectura (54.2\%) y administración (46.7\%) expresan un bajo nivel de desempeño del docente de Técnicas de Estudio.

En cuanto a la dimensión Materiales didácticos, los estudiantes de arquitectura (37.5\%) perciben un alto nivel de desempeño docente. Por su parte, los estudiantes de ingeniería ambiental (53.6\%), psicología (42.9\%) y contabilidad (48.6\%) presentan un nivel medio. No obstante, arquitectura (54.2\%) expresan un bajo nivel de desempeño del docente de Técnicas de Estudio.

La dimensión capacidades pedagógicas del docente son percibidas como altas, de acuerdo a los estudiantes de contabilidad (48.1\%) y marketing (33.3\%). Por su parte, los estudiantes de ingeniería sistemas (45\%) y psicología (42.9\%) presentan un nivel medio. No obstante, arquitectura (58.3\%) y administración (43.3\%) expresan un bajo nivel de desempeño del docente de Técnicas de Estudio.

Finalmente, la dimensión responsabilidad en el desempeño es percibida como alta por los estudiantes de contabilidad (42.9\%) y marketing (33.3\%). Por su parte, los estudiantes de ingeniería sistemas (65\%), ingeniería ambiental (53.6\%) y administración a (40\%) presentan un nivel medio. No obstante, los estudiantes de arquitectura (45.8\%) expresan un bajo nivel de desempeño del docente de Técnicas de Estudio.

\section{Rendimiento académico}

La variable rendimiento académico, se presenta a continuación, considerando las diferentes escuelas profesionales.

Tabla 4

Niveles de promedio

\begin{tabular}{ccccccccc}
\hline Promedio & $\begin{array}{c}\text { Ingeniería } \\
\text { ambiental }\end{array}$ & $\begin{array}{c}\text { Ingeniería } \\
\text { de } \\
\text { sistemas }\end{array}$ & Arquitectura & Psicología & Marketing & Administración & Contabilidad & Total \\
\hline $\begin{array}{c}\text { Muy } \\
\text { deficiente }\end{array}$ & $1(3.6)$ & $2(10)$ & $3(12.5)$ & $1(14.3)$ & $4(19)$ & $4(13.3)$ & $0(0)$ & $15(9.1)$ \\
Deficiente & $0(0)$ & $0(0)$ & $0(0)$ & $0(0)$ & $5(23.8)$ & $0(0)$ & $0(0)$ & $5(3)$ \\
Regular & $9(32.1)$ & $3(15)$ & $2(8.3)$ & $5(71.4)$ & $4(19)$ & $14(46.3)$ & $0(0)$ & 37
\end{tabular}




\begin{tabular}{ccccccccc} 
Bueno & $12(42.9)$ & $9(45)$ & $17(70.8)$ & $1(14.3)$ & $5(23.8)$ & $10(33.3)$ & $21(60)$ & $(45.5)$ \\
Excelente & $6(21.4)$ & $6(30)$ & $2(8.3)$ & 0 & $3(14.3)$ & $2(6.7)$ & $14(40)$ & $33(20)$ \\
Total & $28(100)$ & $20(100)$ & $24(100)$ & $7(100)$ & $21(100)$ & $30(100)$ & $35(100)$ & 165 \\
\hline
\end{tabular}

Fuente: Elaboración propia

En la tabla 04, el $65.5 \%$ de los estudiantes tiene un promedio entre bueno y excelente, siendo los de las escuelas de arquitectura y contabilidad quienes destacan en un promedio bueno; mientras que los de ingeniería de sistemas sobresalen con un promedio excelente.

\section{Prueba de hipótesis}

Prueba de normalidad

Tabla 05

Prueba de Kolmogorov-Smirnov para una muestra

\begin{tabular}{llcc}
\hline & & Promedio & $\begin{array}{c}\text { Desempeño } \\
\text { docente }\end{array}$ \\
\hline $\mathrm{N}$ & & 165 & 165 \\
Parámetros normales $^{\mathrm{a},}$ & Media & 14.29 & 113.29 \\
& Desviación estándar & 3.69 & 21.41 \\
Máximas diferencias & Absoluta & .311 & .101 \\
extremas & Positivo & .202 & .076 \\
& Negativo & -.311 & -.101 \\
Estadístico de prueba & & .311 & .101 \\
Sig. asintótica (bilateral) &, 000 & .000 \\
\hline Fin
\end{tabular}

Fuente: Elaboración propia

a. La distribución de prueba es normal.

b. Se calcula a partir de datos.

El valor de $\mathrm{p}<.05$, entonces los datos no tienen una distribución normal. Por lo tanto, es no paramétrica y se realizará la prueba de correlación de Spearman.

\subsection{Contrastación de la hipótesis general}

$\mathrm{H}_{1}$ : Existe relación significativa entre la percepción del desempeño docente y el rendimiento académico de los estudiantes del curso de técnicas de estudio de una universidad privada confesional, 2018.

$\mathrm{H}_{0}$ : No existe relación significativa entre la percepción del desempeño docente y el rendimiento académico de los estudiantes del curso de técnicas de estudio de una universidad privada confesional, 2018.

\section{Regla de decisión}

Si el $p$ valor $>0,05$ se acepta la Hipótesis Nula. 
Si el $p$ valor $<0,05$ se rechaza la Hipótesis Nula, por lo tanto, se acepta la Hipótesis Alterna.

Tabla 06

Correlación de variable predictora y criterio

\begin{tabular}{lcccc}
\hline & & $\begin{array}{c}\text { Rendimiento } \\
\text { académico }\end{array}$ & $\begin{array}{c}\text { Desempeño } \\
\text { docente }\end{array}$ \\
\hline Rho de Spearman & $\begin{array}{c}\text { Rendimiento } \\
\text { académico }\end{array}$ & $\mathrm{r}$ & 1.000 &, $453^{* *}$ \\
& $\mathrm{p}$ & & .000 \\
Desempeño docente & $\mathrm{N}$ & 165 & 165 \\
& $\mathrm{r}$ &, $453^{* *}$ & 1.000 \\
& $\mathrm{p}$ & .000 & 165 \\
\hline
\end{tabular}

Fuente: Elaboración propia

**. La correlación es significativa en el nivel 0,01 (bilateral).

De acuerdo a la Tabla 06, el p valor es $\mathrm{p}<0.05$, se rechaza la hipótesis nula y se acepta la hipótesis alterna. El valor de r=0.453, de acuerdo a Martínez Rebollar \& Campos Francisco (2015) este valor está en el rango de correlación positiva moderada.

\subsection{Contrastación de hipótesis especificas}

\subsubsection{Primera hipótesis especifica}

$\mathrm{H}_{1}$ : Existe relación significativa entre la percepción de estrategias didácticas y el aprendizaje de los estudiantes del curso de técnicas de estudio de una universidad privada confesional, 2018.

$\mathrm{H}_{0}$ : No existe relación significativa entre la percepción de estrategias didácticas y el aprendizaje de los estudiantes del curso de técnicas de estudio de una universidad privada confesional, 2018.

\section{Regla de decisión}

Si el $p$ valor $>0,05$ se acepta la Hipótesis Nula.

Si el $\mathrm{p}$ valor $<0,05$ se rechaza la Hipótesis Nula, por lo tanto, se acepta la Hipótesis Alterna.

Tabla 07

Correlación de Estrategias didácticas y rendimiento académico

\begin{tabular}{lcccc}
\hline & & $\begin{array}{c}\text { Rendimiento } \\
\text { académico }\end{array}$ & $\begin{array}{c}\text { Estrategias } \\
\text { didácticas }\end{array}$ \\
\hline $\begin{array}{l}\text { Rho de } \\
\text { Spearman }\end{array}$ & $\begin{array}{c}\text { Rendimiento } \\
\text { académico }\end{array}$ & $\mathrm{r}$ & 1.000 & $.404^{* *}$ \\
& $\mathrm{p}$ & $\mathrm{N}$ & 165 & .000 \\
& $\begin{array}{c}\text { Estrategias } \\
\text { didácticas }\end{array}$ & $\mathrm{r}$ & $.404^{* *}$ & 165 \\
& & & & 1.000 \\
\hline
\end{tabular}




\begin{tabular}{cccc} 
& $\mathrm{p}$ & .000 & \\
& $\mathrm{~N}$ & 165 & 165 \\
\hline Fuente: Elaboración propia & &
\end{tabular}

Fuente: Elaboración propia

**. La correlación es significativa en el nivel 0,01 (bilateral).

En la tabla 07, se observa que las estrategias didácticas están relacionadas con el rendimiento académico de los estudiantes. Considerando el Rho Spearman ( $\mathrm{r}=.404)$, Martínez Rebollar \& Campos Francisco (2015) sostienen que este valor está en el rango de correlación positiva moderada.

\section{Segunda hipótesis específica}

$\mathrm{H}_{2}$ : Existe relación significativa entre la percepción de materiales didácticos y el aprendizaje de los estudiantes del curso de técnicas de estudio de una universidad privada confesional, 2018.

$\mathrm{H}_{0}$ : No existe relación significativa entre la percepción de materiales didácticos y el aprendizaje de los estudiantes del curso de técnicas de estudio de una universidad privada confesional, 2018.

\section{Regla de decisión}

Si el $p$ valor $>0,05$ se acepta la Hipótesis Nula.

Si el $\mathrm{p}$ valor $<0,05$ se rechaza la Hipótesis Nula, por lo tanto, se acepta la Hipótesis Alterna.

Tabla 08

Correlación de materiales didácticos y rendimiento académico 


\begin{tabular}{lcccc}
\hline & & $\begin{array}{c}\text { Rendimiento } \\
\text { académico }\end{array}$ & $\begin{array}{c}\text { Materiales } \\
\text { didácticos }\end{array}$ \\
\hline $\begin{array}{l}\text { Rho de } \\
\text { Spearman }\end{array}$ & $\begin{array}{c}\text { Rendimiento } \\
\text { académico }\end{array}$ & $\mathrm{r}$ & 1.000 & $.404^{* *}$ \\
& $\mathrm{p}$ & & .000 \\
& $\mathrm{~N}$ & 165 & 165 \\
& Materiales & $\mathrm{r}$ & $.404^{* *}$ & 1.000 \\
didácticos & $\mathrm{p}$ & .000 & 165 \\
\hline
\end{tabular}

Fuente: Elaboración propia

**. La correlación es significativa en el nivel 0,01 (bilateral).

En la tabla 08, se observa que los materiales didácticos están relacionados con el rendimiento académico de los estudiantes. Considerando el Rho Spearman ( $\mathrm{r}=0.404)$, según establece Martínez Rebollar \& Campos Francisco (2015) este valor está en el rango de correlación positiva moderada. Dónde el $\mathrm{p}(\mathrm{sig}=0.00)$ valor es $\mathrm{p}<0.05$, se rechaza la hipótesis nula y se acepta la hipótesis alterna.

\section{Tercera hipótesis específica}

$\mathrm{H}_{3}$ : Existe relación significativa entre la percepción de capacidades pedagógicas y el aprendizaje de los estudiantes del curso de técnicas de estudio de una universidad privada confesional, 2018.

$\mathrm{H}_{0}$ : No existe relación significativa entre la percepción de capacidades pedagógicas y el aprendizaje de los estudiantes del curso de técnicas de estudio de una universidad privada confesional, 2018.

\section{Regla de decisión}

Si el $p$ valor $>0,05$ se acepta la Hipótesis Nula.

Si el p valor $<0,05$ se rechaza la Hipótesis Nula, por lo tanto, se acepta la Hipótesis Alterna.

Tabla 09

Correlación de capacidades padagógicas y rendimiento académico

\begin{tabular}{|c|c|c|c|c|}
\hline & & & $\begin{array}{l}\text { Rendimiento } \\
\text { académico }\end{array}$ & $\begin{array}{l}\text { Capacidades } \\
\text { pedagógicas }\end{array}$ \\
\hline \multirow[t]{6}{*}{$\begin{array}{l}\text { Rho de } \\
\text { Spearman }\end{array}$} & \multirow{3}{*}{$\begin{array}{l}\text { Rendimiento } \\
\text { académico }\end{array}$} & $\begin{array}{l}\text { Coeficiente de } \\
\text { correlación }\end{array}$ & 1.000 & $.442^{* *}$ \\
\hline & & Sig. (bilateral) & & .000 \\
\hline & & $\mathrm{N}$ & 165 & 165 \\
\hline & \multirow{3}{*}{$\begin{array}{l}\text { Capacidades } \\
\text { pedagógicas }\end{array}$} & $\begin{array}{l}\text { Coeficiente de } \\
\text { correlación }\end{array}$ & $.442^{* *}$ & 1.000 \\
\hline & & Sig. (bilateral) & .000 & \\
\hline & & $\mathrm{N}$ & 165 & 165 \\
\hline
\end{tabular}

Fuente: Elaboración propia

**. La correlación es significativa en el nivel 0,01 (bilateral) 
En la tabla 09, se observa que las capacidades pedagógicas están relacionadas con el rendimiento académico de los estudiantes. Considerando el Rho Spearman ( $\mathrm{r}=0.442)$, Martínez Rebollar \& Campos Francisco (2015) este valor está en el rango de correlación positiva moderada. Dónde el $p(\operatorname{sig}=.00)$ valor es $p<0.05$, se rechaza la hipótesis nula y se acepta la hipótesis alterna.

\section{Cuarta hipótesis específica}

$\mathrm{H}_{4}$ : Existe relación significativa entre la percepción de responsabilidad del docente y el aprendizaje de los estudiantes del curso de técnicas de estudio de una universidad privada confesional, 2018.

$\mathrm{H}_{0}$ : No existe relación significativa entre la percepción de responsabilidad del docente y el aprendizaje de los estudiantes del curso de técnicas de estudio de una universidad privada confesional, 2018.

\section{Regla de decisión}

Si el $p$ valor $>0,05$ se acepta la Hipótesis Nula.

Si el p valor $<0,05$ se rechaza la Hipótesis Nula, por lo tanto, se acepta la Hipótesis Alterna.

Tabla 10

Correlación de responsabilidad en el desempeño docente y rendimiento académico

\begin{tabular}{lcccc}
\hline & & $\begin{array}{c}\text { Rendimiento } \\
\text { académico }\end{array}$ & $\begin{array}{c}\text { Responsabilidad en } \\
\text { el desempeño }\end{array}$ \\
\hline $\begin{array}{l}\text { Rho de } \\
\text { Spearman }\end{array}$ & $\begin{array}{c}\text { Rendimiento } \\
\text { académico }\end{array}$ & $\begin{array}{c}\text { Coeficiente de } \\
\text { correlación } \\
\text { Sig. (bilateral) }\end{array}$ & 1.000 & $.447^{* *}$ \\
& $\begin{array}{c}\mathrm{N} \\
\text { Responsabilidad en } \\
\text { el desempeño }\end{array}$ & $\begin{array}{c}\text { Coeficiente de } \\
\text { correlación } \\
\text { Sig. (bilateral) }\end{array}$ & .465 & .000 \\
& $\mathrm{~N}$ & .000 & 165 \\
& & 165 & 1.000 \\
\hline
\end{tabular}

Fuente: Elaboración propia

**. La correlación es significativa en el nivel 0,01 (bilateral).

En la tabla 10, se observa que la responsabilidad en el desempeño está relacionada con el rendimiento académico de los estudiantes. Considerando el Rho Spearman $(\mathrm{r}=.447)$, la correlación es directa y significativa. Dónde el $\mathrm{p}(\mathrm{sig}=.00)$ valor es $\mathrm{p}<.05$, se rechaza la hipótesis nula y se acepta la hipótesis alterna.

\section{Discusión}


El objetivo principal de la investigación fue determinar si existe relación significativa entre la percepción del desempeño docente y el rendimiento académico de los estudiantes del curso de técnicas de estudio de una universidad privada confesional, 2018. Se identificó que existe correlación entre el desempeño docente y el rendimiento académico, resultados similares fueron obtenidos por Piña (2010), Gómez López, Rosales Gracia, García Galaviz, \& Berrones Sánchez (2011), Navarrete et al., (2012), Maldonado (2012), Palomino Zamudio (2012), Arraiza Alvarado (2015), La Serna Studzinski, Becerra Marsano, \& Hongrui Zhang (2016), Cerda et al. (2017)

El primer objetivo específico fue determinar si existe relación significativa entre la percepción de estrategias didácticas y el rendimiento académico de los estudiantes del curso de técnicas de estudio de una universidad privada confesional, 2018. Los resultados demuestran que existe relación significativa entre la percepción de estrategias didácticas y el aprendizaje. Resultado similar al obtenido por Palomino Zamudio (2012). Por su parte, Gómez López et al. (2011) destaca que las estrategias de enseñanza aprendizaje activo participativas favorecen el autoestudio con un enfoque contextual, propician el aprendizaje significativo; de ahí que se propone que, a mayor calificación de la práctica docente, mayor rendimiento académico. Contrario a lo identificado por Navarrete et al., (2012), quien menciona que existe una asociación inversa entre rendimiento académico y estrategias de aprendizaje. Cerda et al. (2017), argumenta que es importante que los profesionales (docentes) incorporen a sus competencias pedagógicas, un amplio número de estrategias y recursos didácticos orientados a promover el desarrollo de competencias.

El segundo objetivo específico fue determinar si existe relación significativa entre la percepción de materiales didácticos y el rendimiento académico de los estudiantes del curso de técnicas de estudio de una universidad privada confesional, 2018. Los resultados demuestran que existe relación significativa entre la percepción de materiales didácticos y el aprendizaje de los estudiantes. Como lo hace notar Maldonado (2012) y Palomino Zamudio (2012), cuanto más el docente que emplee medios didácticos, mayor será el aprendizaje. Pérez (2015) sugiere que se requiere un mejoramiento continuo de los materiales didácticos, mientras que Barriga Rodriguez (2016) ratifica que al trabajar en conjunto las estrategias, medios y materiales didácticos, se aporta calidad a la docencia en la universidad.

El tercer objetivo específico fue determinar si existe relación significativa entre la percepción de capacidades pedagógicas y el rendimiento académico de los estudiantes del 
curso de técnicas de estudio de una universidad privada confesional, 2018. Se demostró que existe relación significativa entre la percepción de capacidades pedagógicas y el aprendizaje de los estudiantes. Los resultados concuerdan con los datos obtenidos por Palomino Zamudio (2012), a mayor dominio del contenido del curso, entonces será mayor el aprendizaje. Para Valdez (citado en Barriga Rodriguez, 2016), la evaluación de capacidades pedagógicas motiva a mejorar el desempeño del docente.

El cuarto objetivo específico fue determinar si existe relación significativa entre la percepción de responsabilidad del docente y el rendimiento académico de los estudiantes del curso de técnicas de estudio de una universidad privada confesional, 2018. Se evidencia que existe relación significativa entre la percepción de responsabilidad del docente y el aprendizaje de los estudiantes. Resultados similares describen Maldonado (2012) y Palomino Zamudio (2012), "cuando el docente asiste puntualmente, desarrolla los temas de acuerdo al silabo con un ordenamiento metodológico", se alcanza un mayor aprendizaje.

De igual modo, Barriga Rodriguez (2016) alude que la responsabilidad del docente es trascendental para propiciar el aprendizaje, por ende, el rendimiento de los estudiantes. Piña (2010), indica que la responsabilidad docente es el conjunto de actitudes que propician procesos de calidad de enseñanza.

\section{Conclusiones}

Después de realizar el análisis de la investigación titulada "Percepción del desempeño docente y su relación con el rendimiento académico de los estudiantes del curso de técnicas de estudio de una universidad privada confesional, 2018”, se concluye que:

1. Existe relación significativa entre la percepción del desempeño docente y el rendimiento académico de los estudiantes del curso de técnicas de estudio de una universidad privada confesional, 2018. La correlación es correlación positiva moderada $(r=0.453 ; p=0.00)$. El 62.4\% considera que el desempeño del docente del curso de técnicas de estudio está entre bueno y excelente, lo cual se ve reflejado en que el $65.5 \%$ de los estudiantes tiene un promedio superior a 15 , lo que equivale a que $20 \%$ de los estudiantes tiene un promedio excelente, el $45.5 \%$ es bueno.

2. Existe relación significativa entre la percepción de estrategias didácticas y el aprendizaje de los estudiantes del curso de técnicas de estudio de una universidad privada confesional, 2018. La correlación es correlación positiva moderada $(r=0.404 ; p=0.00)$. 
3. Existe relación significativa entre la percepción de materiales didácticos y el aprendizaje de los estudiantes del curso de técnicas de estudio de una universidad privada confesional, 2018. La correlación es correlación positiva moderada $(r=0.404 ; p=0.00)$.

4. Existe relación significativa entre la percepción de capacidades pedagógicas y el aprendizaje de los estudiantes del curso de técnicas de estudio de una universidad privada confesional, 2018. La correlación es correlación positiva moderada $(r=0.442 ; p=0.00)$.

5. Existe relación significativa entre la percepción de responsabilidad del docente y el aprendizaje de los estudiantes del curso de técnicas de estudio de una universidad privada confesional, 2018. Correlación positiva moderada $(\mathrm{r}=0.447 ; \mathrm{p}=0.00)$.

\section{Recomendaciones}

1. Brindar capacitación continua a los docentes, a fin de potenciar las estrategias didácticas que emplean con los estudiantes, garantizando que el docente se convierta en un experto en la materia. Considerando que las necesidades académicas deben ser suplidas acorde a los cambios tecnológicos y de desarrollo.

2. Fomentar actividades de fidelización docente, considerando que con el fortalecimiento de su compromiso aumentará su satisfacción en el desempeño de la práctica docente.

3. Evaluar otros factores que pueden intervenir en el rendimiento académico de los estudiantes. Considerando que trabajar y estudiar, modo de pago de estudios pueden ser algunos de los condicionantes externos del rendimiento académico.

4. Respecto a los estudiantes que presentan un bajo rendimiento académico, se requiere un seguimiento personalizado para identificar qué factores afectan su rendimiento y potenciar sus capacidades, logrando mejorar su desempeño. Además de comparar su desempeño respecto a otros cursos, para evidenciar que necesidades de aprendizaje tiene el estudiante.

5. Se propone realizar una investigación cualitativa, utilizando el método de focus group, para recabar las opiniones tienen los estudiantes respecto al desempeño docente y como este afecta su rendimiento académico.

\section{Referencias}

Alterio Ariola, H. G., \& Pérez Loyo, A. H. (2009). Evaluación de la función docente según el desempeño de los profesores y la opinión estudiantil. Educación Médica Superior, 23(3), 1-14. Retrieved from https://web.a.ebscohost.com/ehost/pdfviewer/pdfviewer?vid=4\&sid=3d7ed01e-c94e4f40-b74c-280ef3a0c557\%40sessionmgr4010

Aparicio, S., Muñoz, B., Robson, C., Ruiz, L., Secreto, M., Sepliarsky, P., \& Escobar, M. (2011, November). El rendimiento de los estudiantes. Universatil. 
Arraiza Alvarado, J. J. (2015). Formación profesional permanente, desempeño profesional del docente y rendimiento académico de los estudiantes del IV, VI, VIII y X ciclo de estudios Facultad de Educación Universidad Nacional Mayor de San Marcos 2013-2. Universidad Nacional Mayor de San Marcos. Maestría Facultad de Educación. Retrieved from http://cybertesis.unmsm.edu.pe/handle/cybertesis/5624

Barriga Rodriguez, R. D. (2016). Clima organizacional y desempeño docente en la universidad "Jaime Bausate y Meza” Jesús María - Lima, 2016. Tesis Digitales UNMSM, 172.

Cerda, G., Pérez, C., Aguilar, M., Aragón, E., Cerda, G., Pérez, C., ... Aragón, E. (2017). Algunos factores asociados al desempeño académico en matemáticas y sus proyecciones en la formación docente. Educação E Pesquisa, 44(0). https://doi.org/10.1590/s16784634201706155233

Fernandes, D. J., Sotolongo, M., Ii, S., Cristóbal, C., \& Martínez, M. (2016). Procedimiento para la evaluación del desempeño del personal docente en las universidades públicas angolanas Procedure for the evaluation of the teaching performance in the public angolan universities, $X X X V I I(2), 178-189$.

Garbanzo, M. (2007). Factores asociados al rendimiento académico en estudiantes universitarios, una reflexión desde la calidad de la educación superior pública. Revista de Educación, 31(1), 43-63.

Gómez López, V. M., Rosales Gracia, S., García Galaviz, J. L., \& Berrones Sánchez, K. (2011). Correlación entre la práctica docente y rendimiento académico en un grupo de estudiantes de medicina Correlation between teaching practice and academic performance in a group of medical students. Archivos En Artículo Original, 13(3), 117121. Retrieved from http://www.medigraphic.com/pdfs/medfam/amf-2011/amf113c.pdf

Hernandez, R., Fernández, C., \& Baptista, L. (2010). Metodologia de la Investigacion (5th ed.). Mexico: Mc Graw Hill.

La Serna Studzinski, K., Becerra Marsano, A., \& Hongrui Z. (2016). La relación de las encuestas de evaluación docente con el rendimiento académico: La evidencia empírica en la Universidad del Pacífico. Revista Iberoamericana de Evaluación Educativa, 7(2), 105-115. Retrieved from http://eds.b.ebscohost.com/eds/pdfviewer/pdfviewer?vid=1\&sid=a702cd4c-23b6-4fa5a0b0-20882caa477b\%40pdc-v-sessmgr02

Maldonado, R. (2012). Percepción del desempeño docente en relación con el aprendizaje de los estudiantes. Universidad San Martín de Porres.

Martínez Rebollar, A., \& Campos Francisco, W. (2015). The Correlation Among Social Interaction Activities Registered Through New Technologies and Elderly's Social Isolation Level. Revista Mexicana de Ingeniería Biomédica, 36(3), 177-188. https://doi.org/10.17488/RMIB.36.3.4

Navarrete, B., Inostroza, A., Yesenia, S., Muñoz, M., Villalobos, C., Evelyn, V., ... Patricia, S. (2012). Relación entre rendimiento académico, estrategias de aprendizaje, motivación y estrés en estudiantes de enfermería de la universidad del Biobío, 2012. Universidad del Biobio. Retrieved from http://repobib.ubiobio.cl/jspui/bitstream/123456789/782/1/Baeza Navarrete\%2C Aidee.pdf

Ortega, M. del C. (2010). Teacher's emerging competences as a demand of the European 
Higher Education Area. Revista Española de Educación Comparada, O(16), 305-328. Retrieved from http://revistas.uned.es/index.php/REEC/article/view/7534/7202

Ortiz, Y. G., Ortiz, Y. G., Machado, D. L. de C., \& Frutos, O. R. (2014). Estudiantes universitarios con bajo rendimiento académico, ¿qué hacer? EDUMECENTRO, 6(2), $272-278$.

Palomino Zamudio, F. (2012). El desempeño docente y el aprendizaje de los estudiantes de la Unidad Académica de Estudios Generales de la Universidad de San Martín de Porres. Universidad Nacional Mayor de San Marcos.

Pérez, A. C. (2015). Contribución de la evaluación de desempeño al desarrollo profesional del docente universitario. Ciencias Sociales Y Humanas, VI(1), 72-87. https://doi.org/10.15658

Piña, R. (2010). El desempeño docente y su relación con las habilidades del estudiante y el rendimiento académico en la universidad particular de Iquitos, año 2010. Universidad Nacional Mayor de San Marcos. Retrieved from http://cybertesis.unmsm.edu.pe/bitstream/cybertesis/2366/1/Piña_sr.pdf

Ramírez Sánchez, C. (2014). El Aprendizaje Basado en Problemas: estrategia didáctica que fortalece el p...: EBSCOhost. Papeles, 6(11), 61-71. Retrieved from https://web.b.ebscohost.com/ehost/pdfviewer/pdfviewer?vid=1\&sid=7e1f837e-9cbf40b9-86d7-9a28f9cbe96f\%40sessionmgr103 